\title{
Development of Virtual Instrument for Uniaxial Compression Testing of Rock Samples
}

\author{
D. Kuhinek, I. Zorić, P. Hrženjak \\ Faculty of Mining, Geology and Petroleum Engineering, University of Zagreb, Pierottijeva, 6, HR-10002, Zagreb, Croatia, \\ dalibor.kuhinek@rgn.hr, igor.zoric@rgn.hr, petar.hrzenjak@rgn.hr \\ This paper presents the virtual instrument for measurement and determination of uniaxial compression strength and rock \\ sample deformability. It analyzes properties and limitations of several implemented virtual instruments. Furthermore, comparison \\ of implemented virtual instrument properties is presented.
}

Keywords: Uniaxial strength, virtual instrument, measurement system properties

\section{INTRODUCTION}

$\mathrm{D}$ ETERMINATION of rock samples' mechanical properties in laboratory includes preparation of samples with specific dimensions and application of stress according to the chosen test method, [1], [2]. Simultaneously, certain physical quantities are measured using measurement systems which provide complete solution (hardware plus software). The user generally does not have a possibility to change components or functionality of the measurement system. For the research of test methods for rock samples, a measurement system, which enables full flexibility in development of measuring methods, is designed and implemented. The measurement system consists of measurement equipment and software (Labview) which controls the test. To ensure confidence in measurement results obtained by this measurement system, properties and limitations of implemented systems are analyzed.

The basic test for determining mechanical properties which is conducted on rock samples is uniaxial compression strength and deformability of the material. According to the Suggested Methods of the International Society for Rock Mechanics, the constant increasing stress in the range of 0.5 $\mathrm{MPa} / \mathrm{s}$ to $1 \mathrm{MPa} / \mathrm{s}$ is applied to the sample, with minimum diameter of $54 \mathrm{~mm}$ and height of 2.5 to 3 times larger than its diameter, until disintegration. In this test it is necessary to measure force and sample axial deformation. Results obtained by the test are maximum stress prior to disintegration and Young's modulus of elasticity. If diametral deformations are measured, Poisson's coefficient is also obtained.

\section{MEASUREMENT SYSTEM}

The measurement chain in Fig.1 is assembled from a transducer for each specified quantity, connecting cables, conditioning module and measurement card for digitalization and data acquisition. The system uses several analog input channels (AI) and two analog output channels (AO). Pressure transducer uses strain gauges model Controls $82-\mathrm{P} 0354^{1)}$ which measures pressure from 0 bar to 700 bar. For measurement of axial deformations three LVDTs Schaewitz MHR050 ${ }^{1)}$ and three LVDT conditioning modules LVC-2500 ${ }^{1)}$ are used.

The used compression machine is Soiltest CT-732 with hydraulic pump, high pressure valve in the servo device and three-phase motor that drives the pump.

Table 1 lists components used for stress measurement chain implementation (A to E). Each of the listed elements has producer defined properties which will influence complete measurement chain properties and subsequently the result quality. Data acquisition card is NI PCI 6024 E.

Tab.1. Equipment used for various implementations of stress measurement chain.

\begin{tabular}{|c|l|}
\hline & \multicolumn{1}{|c|}{ Conditioning } \\
\hline A & $\begin{array}{l}\text { NI SC-2043-SG }{ }^{1)} \text { strain gauge conditioning } \\
\left(U_{\text {ex }}=2.5 \mathrm{~V}\right)\end{array}$ \\
\hline B & $\begin{array}{l}\text { NI SC-2345 }{ }^{1)} \text { with module SCC-SG04 } \\
\left(U_{\text {ex }}=2.5 \text { V }\right) \text { and SCC-AI13 }\end{array}$ \\
\hline C & $\begin{array}{l}\text { NI SC-2345 with module SCC-SG24 } \\
\left(U_{\text {ex }}=10 \text { V }\right) \text { and SCC-AI13 module }\end{array}$ \\
\hline D & $\begin{array}{l}\text { NI SCB-68 }{ }^{1)} \text { connection block with SG } \\
\text { external excitation }\left(U_{\text {ex }}=2.5 \text { V }\right)\end{array}$ \\
\hline E & $\begin{array}{l}\text { NI SCB-68 connection block with SG external } \\
\text { excitation }\left(U_{\text {ex }}=10 \text { V }\right)\end{array}$ \\
\hline
\end{tabular}

\section{NOISE AND ACCURACY OF THE MEASUREMENT SYSTEM}

Two basic parameters that influence the measurement system and its quality are noise and accuracy. Noise influences the control of measurement system and uncertainty of measured quantity.

${ }^{1}$ Brand names are used for the purpose of identification. Such use does not imply endorsement by authors and it does not assume that the equipment is the best available. 


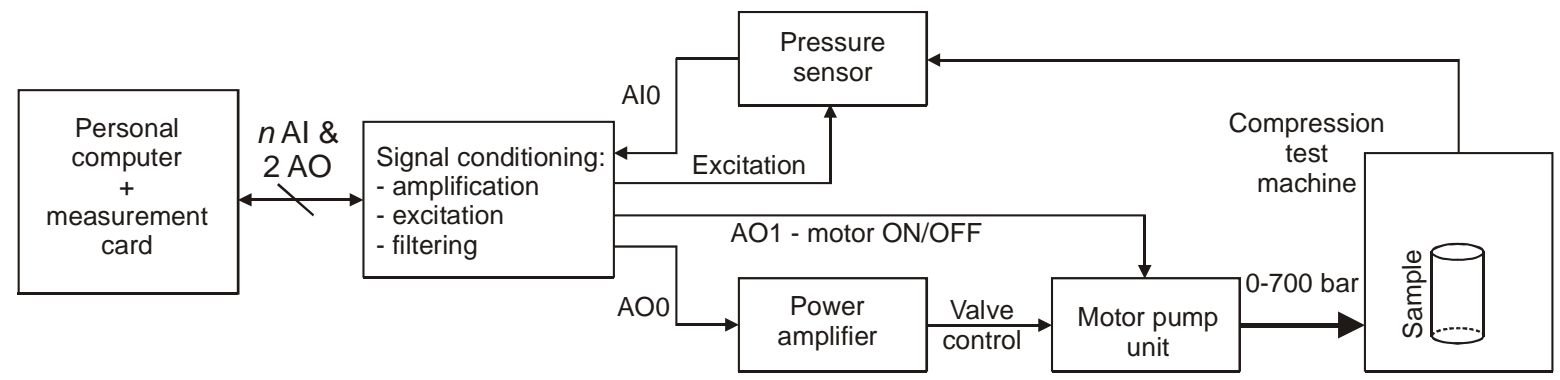

Fig.1. Components of hardware part of the virtual instrument for measurement of rock samples' deformations.

\section{A. System noise}

Overall noise depends on the noise of individual components, in this case noise of strain gauges $\sigma_{\mathrm{SG}}$, cable noise $\sigma_{\mathrm{Cab}}$ and preamplifier input noise $\sigma_{\mathrm{Cond}}$. Under the assumption that all of this noise is Gaussian white noise, overall RMS noise $\sigma$ is determined according to (1):

$$
\sigma=\sqrt{\sigma_{\mathrm{SG}}^{2}+\sigma_{\mathrm{Cab}}^{2}+\sigma_{\mathrm{Cond}}^{2}}
$$

This noise is amplified by amplifier gain $A$ and then the signal is added to measurement card input noise $\sigma_{\mathrm{DAQ}}$. Overall RMS noise $\sigma_{\text {NoiseIn }}$, as seen from input is calculated according to (2).

$$
\sigma_{\text {Noise In }}=\frac{\sqrt{\left(A \sqrt{\sigma_{\mathrm{SG}}^{2}+\sigma_{\mathrm{Cab}}^{2}+\sigma_{\mathrm{Cond}}^{2}}\right)^{2}+\left(\sigma_{\mathrm{DAQ}}\right)^{2}}}{A}
$$

Thermal noise of $350 \Omega$ strain gauge, at room temperature and with bandwidth of $1 \mathrm{~Hz}$, is approximately $2.4 \mathrm{nV}$. With a hundred times larger bandwidth, noise voltage is ten times greater.

Cable noise can be lowered using grounded shielded cable and using cables as short as possible. Input noise of conditioning modules is listed in Tab. 2 and is in range of a few microvolts.

Tab.2. Input noise of available modules.

\begin{tabular}{|l|l|}
\hline Module & Input noise (manufacturer limit) (rms V) \\
\hline $\mathrm{A}$ & $5 \mu \mathrm{V}$ (low pass filter $1.6 \mathrm{kHz}$ ) \\
\hline $\mathrm{B}$ & $1 \mu \mathrm{V}$ (low pass filter $1.6 \mathrm{kHz}$ ) \\
\hline $\mathrm{C}$ & $3 \mu \mathrm{V}$ (low pass filter $1.6 \mathrm{kHz}$ ) \\
\hline $\mathrm{D} \& \mathrm{E}$ & $\begin{array}{l}63 \mu \mathrm{V} \text { (bandwidth } 225 \mathrm{kHz} \text { ) } \\
\text { (for smallest range } \pm 50 \mathrm{mV} \text { ) }\end{array}$ \\
\hline
\end{tabular}

Under the assumption that strain gauge and cable noise are negligible, follows (3):

$$
\sigma_{\text {NoiseIn }}=\sqrt{\sigma_{\text {Cond }}^{2}+\left(\frac{\sigma_{\mathrm{DAQ}}}{A}\right)^{2}}
$$

The analysis of noise contributions shows that in the used measurement chain the largest noise contribution comes from the first electronic component $\left(\sigma_{\text {NoiseIn }} \approx \sigma_{\text {Cond }}\right)$, i.e., the preamplifier, if used, or measurement card if the preamplifier is not used $\left(\sigma_{\text {NoiseIn }}=\sigma_{\mathrm{DAQ}}\right)$.

If more samples are acquired and mean value is calculated then input noise is divided by square root from the number of samples. Considering that noise of electronic components consists from white and 1/f noise, there is a limit to which faster sampling and averaging makes sense.

\section{B. Accuracy}

Accuracy of the measurement chain is influenced by component properties. Measurement signal of strain gauge based sensor is output voltage $U_{\mathrm{o}}$ in the range of millivolts. This voltage is divided by excitation voltage $U_{\text {ex }}$ (usually $2.5 \mathrm{~V}$ to $10 \mathrm{~V}$ ) to obtain output quantity in volts of output voltage per volt of excitation $(\mathrm{V} / \mathrm{V})$. The result is output quantity in volts proportional to input quantity (e.g., pressure). This also assures that changes in excitation voltage during test will not cause errors.

Preamplifier error limits $G_{\text {Cond }}$ with gain of $A$ connected to the measurement card with error limits $G_{\mathrm{DAQ}}$ give total error limits of output voltage $G_{\mathrm{o}}$, which as seen from input, is determined by (4):

$$
G_{\mathrm{o}}=\sqrt{G_{\mathrm{Cond}}^{2}+\left(\frac{G_{\mathrm{DAQ}}}{A}\right)^{2}}
$$

This means that in the total error, substantial contribution comes from signal conditioning module preamplifier error. The gain of ten or hundred means ten or hundred times smaller contribution of data acquisition which becomes negligible $\left(G_{\mathrm{o}} \approx G_{\text {Cond }}\right)$.

Errors $G_{\text {Cond, }} G_{\text {DAQ }}$ and error of excitation voltage $G_{\text {ex }}$ are calculated by linear addition of all contributions [3], [4] which influences the accuracy of individual system components and then calculated errors are divided by root square of number three to obtain uncertainty of type B [5] which is calculated from data given by manufacturer. Contributions of preamplifier are gain error, offset error, nonlinerity and input noise.

Combined standard uncertainty $u_{\mathrm{c}}$ of voltage ratio $U_{\mathrm{o}} / U_{\mathrm{ex}}$ can be calculated by using uncertainty of output voltage $u\left(U_{\mathrm{o}}\right)$ and uncertainty of excitation voltage $u\left(U_{\mathrm{ex}}\right)$ and is calculated according to GUM [5] which gives (5). 
$u_{\mathrm{c}}\left(\frac{U_{\mathrm{o}}}{U_{\mathrm{ex}}}\right)=\sqrt{\left(\frac{1}{U_{\mathrm{ex}}}\right)^{2} \cdot u^{2}\left(U_{\mathrm{o}}\right)+\left(-\frac{U_{\mathrm{o}}}{U_{\mathrm{ex}}^{2}}\right)^{2} \cdot u^{2}\left(U_{\mathrm{ex}}\right)}$

Expected voltage ratio combined standard uncertainty is calculated for different conditioning modules used in stress measurement chain and presented in Fig.2. The $y$ ordinate represents combined standard uncertainty in dependence to measured voltage ratio. Analysis is performed for modules $A$ to $C$ which include the measurement card and $\mathrm{D}$ and $\mathrm{E}$ for measurement card only. For implementations $\mathrm{C}$ and $\mathrm{E}$, excitation voltage is $10 \mathrm{~V}$. Analysis is performed with the assumption that voltage ratio is $800 \mu \mathrm{V} / \mathrm{V}$, i.e., by using excitation of $2.5 \mathrm{~V}$ giving $2 \mathrm{mV}$ of output voltage or $10 \mathrm{~V}$ giving $8 \mathrm{mV}$ output voltage.

The analysis was made under assumption of 100 samples averaging, with temperature that has not changed more than $1{ }^{\circ} \mathrm{C}$ from last internal calibration, and $10^{\circ} \mathrm{C}$ from last external calibration.

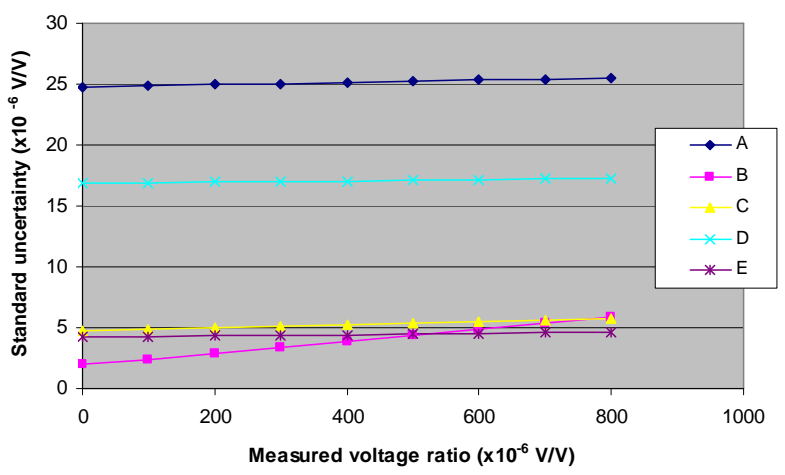

Fig.2. Influence of conditioning module properties and excitation voltage to standard uncertainty of a voltage ratio.

Several conclusions can be reached from this analysis. The increase of excitation voltage $w$ times lowers uncertainty $w$ times. The error of conditioning module gives the largest contribution to measurement uncertainty. Measurement of output voltage without signal conditioning module can give lower measurement uncertainty. In this configuration, overvoltage that can occur can directly reach the measurement card and cause damage to the more expensive component of the measurement system. In addition, overall noise is larger and resolution is smaller. For the first conditioning module (A) dominant contribution to uncertainty is nonlinearity of preamplifier.

Transducer properties also contribute to the measurement chain uncertainty. Usually, properties of electronic components are substantially better than transducer properties and the transducer uncertainty is dominant but, as presented, the electronic part can also have substantial contribution to the overall uncertainty. The conditioning module A has standard uncertainty of $25 \mu \mathrm{V} / \mathrm{V}$ which compared to $800 \mu \mathrm{V} / \mathrm{V}$ is over $3 \%$ and even larger for voltage ratio smaller than $800 \mu \mathrm{V} / \mathrm{V}$.

\section{VIRTUAL INSTRUMENT}

The virtual instrument (VI) is programmed in Labview which enables integration of the measurement system to measure and control the test procedure. The front panel of this VI is divided in three tabs that allow interface to the user. The first tab is designated for input of sample dimensions, has a switch for manual control of the motor pump, a slider for manual valve control and measured values that can be checked before the test starts. The second tab is designated for the input of stress increase in $\mathrm{MPa} / \mathrm{s}$. The third tab has a switch for tare and resetting the time counter, a second switch that enables automatic control and diagrams that represent stress over time and stress to strain dependence. Also, calculated and measured force and stress variables are present. Fig. 3 presents the first tab of the front panel to input sample data and Fig.4 presents the third tab.

The block diagram has DAQmx functions for channel and timing setup, start of data acquisition and a while loop which contains the rest of the program code.

The smallest range of the measurement card is used for the measurement of bridge output voltage and for other channels the range of $\pm 5 \mathrm{~V}$ or $\pm 10 \mathrm{~V}$ is used.

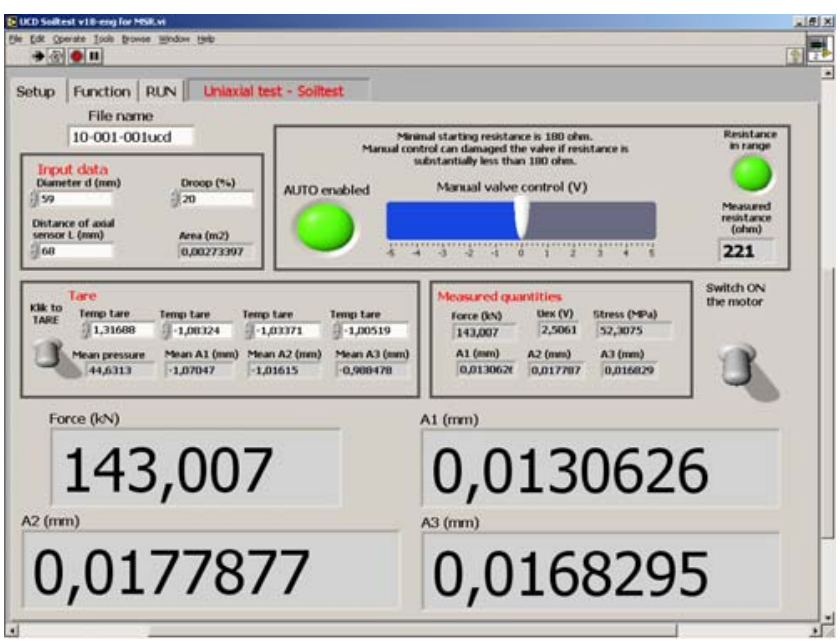

Fig.3. First tab of virtual instrument.

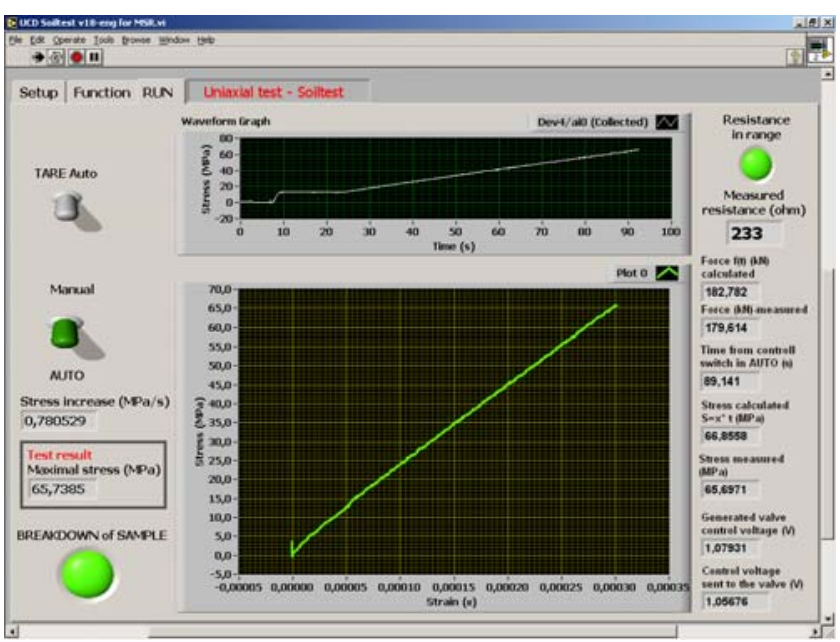

Fig.4. Third tab of virtual instrument. 
The samples are acquired with sample rate of $8 \mathrm{kHz}$ per channel in blocks of 800 samples what together with sample compression function (averaging) gives the effective sample rate of $10 \mathrm{~S} / \mathrm{s}$ per channel. The used measurement card has one $\mathrm{A} / \mathrm{D}$ converter and multiplexer for 16 input channels. The samples are collected from set channels, 800 samples from the first channel, then 800 samples from the second channel and consecutively from the remaining channels. The channels that are not set up are skipped. The described data acquisition consequently leads to ghosting effect, i.e., certain amount of channel signal can appear in the next channel signal. This is especially present if small signal samples on smaller range follow the acquisition of large signal on higher range, [6]. In the presented application, this occurs when acquisition comes to the last channel and then starts again from the first channel. The cause of ghosting effect is charge on the sample and hold capacitor, which can not be discharged fast enough. The recommended solution is to use one shorted channel that is placed in the list of channels prior to the affected channels. This enables fast discharge of the $\mathrm{S} / \mathrm{H}$ capacitor [6]. The second solution is to repeat acquisition on the channel with smaller signal until ghosting effect becomes substantially small. The number of repetitions should be experimentally verified.

The ghosting identification experiments were performed for module A and B. The samples of output voltage are acquired three times and then excitation voltage samples are acquired. This is repeated in a loop. The result is that in conditioning module A there is no substantial error caused by ghosting. Module B, however, gives error of $4 \%$ in the first measurement when compared to the second and the third measurement which proves that ghosting is present and it should be taken into account.

After averaging, the channels are separated into wires that lead the data to functions of VI where they are necessary, such as tare section, diagrams and data saving function, the valve position and control generation section.

For control, two analog outputs are used, the first gives voltage for valve motor drive and the second for switching on and off pump motor. Output samples are written once per loop, i.e., $10 \mathrm{~S} / \mathrm{s}$.

Fig.5 presents stress change which can be achieved with the used measurement system using control voltage in range from $1 \mathrm{~V}$ to $9 \mathrm{~V}$.

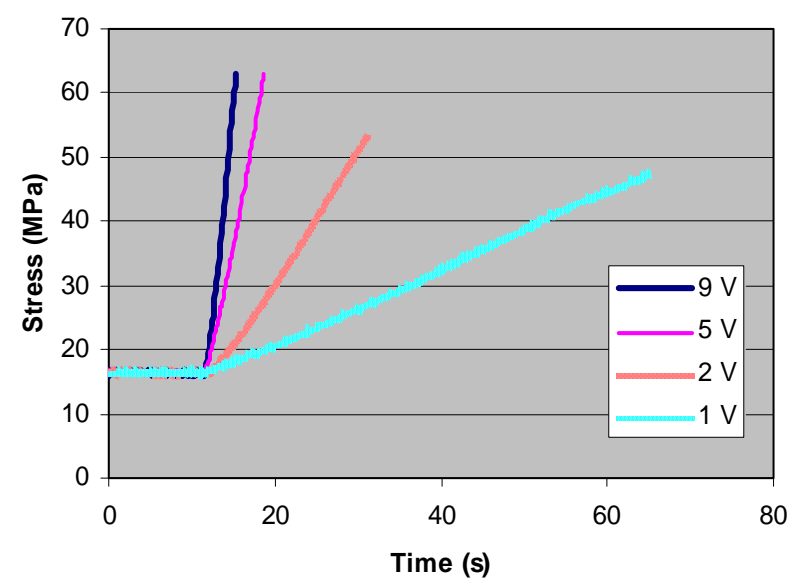

Fig.5. Influence of valve voltage to the increase of stress.
Table 3 presents stress increase in dependence to control voltage. The valve voltage can be up to $10 \mathrm{~V}$, which leads to stress increase of $15 \mathrm{MPa} / \mathrm{s}$. It is obvious that the system can achieve the desired stress increase.

Tab.3. Increase of stress in dependence to the valve control voltage.

\begin{tabular}{|c|c|}
\hline Valve voltage $(\mathrm{V})$ & Increase of stress $(\mathrm{MPa} / \mathrm{s})$ \\
\hline 1 & 0.6 \\
\hline 2 & 2 \\
\hline 5 & 6.6 \\
\hline 9 & 13.3 \\
\hline
\end{tabular}

To achieve the increase of stress of $0.75 \mathrm{MPa} / \mathrm{s}$, the control voltage has to be approximately $1.3 \mathrm{~V}$. The valve control voltage $U_{\text {valve }}$ is generated as function of difference between measured and calculated stress $S$ which depends on time passed from the start of the test, i.e., from the start of automatic control. The control voltage is generated according to (6). The generated voltage is connected to power amplifier that drives the valve motor.

$$
U_{\text {valve }}=-0.5 \cdot\left(S_{\text {measured }}-S_{\text {calculated }}\right)+0.5(\mathrm{~V})
$$

For more complex functions of stress increase it is necessary to optimize and verify the control signal generation. Use of PID controller is recommended.

The code of VI also has a protection mechanism for compression test machine at the sample disintegration range. It is activated if the calculated stress is more than $20 \%$ larger than the measured stress and causes the pump motor to switch off and the valve control voltage is set to zero. This mechanism is turned off for the short period of time at the beginning of the test so that noise does not trigger it off. The last protection is achieved by a limiting switch that triggers if the cylinder of the testing machine moves too much.

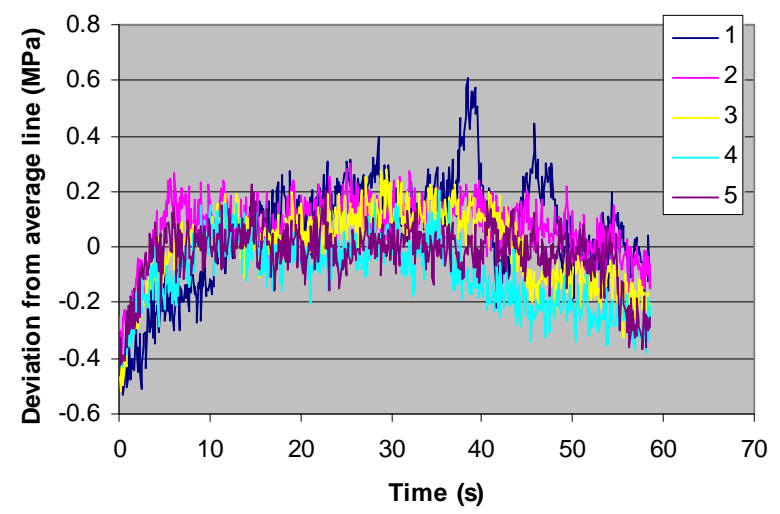

Fig.6. Result from five consecutive tests with module A.

The complete measurement system was tested using a metal sample with dimensions similar to the rock samples to be tested. The module A and B was used in this test. The test was repeated five times up to the stress of $60 \mathrm{MPa}$ and then the testing was aborted. The data was analyzed and linear regression line parameters were calculated for each test. 
Average slope and intercept was used to draw difference between individual test and average line presented in Fig.6 and Fig.7. The stress was in the range of $\pm 0.6 \mathrm{MPa}$ for both cases. Average slope was $0.76 \mathrm{MPa}$ with standard deviation of $0.4 \%$ for module $\mathrm{A}$ and $0.6 \%$ for module $\mathrm{B}$. This is sufficient for this application.

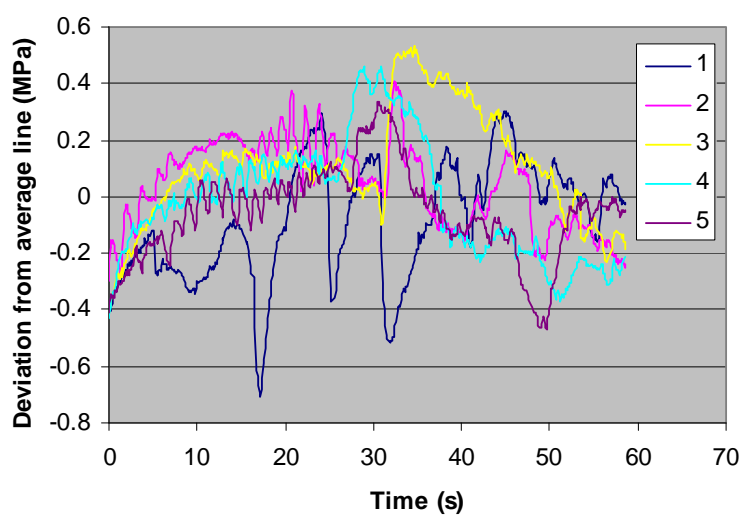

Fig.7. Result from five consecutive tests with module B.

Use of module B gives better uncertainty but the resulting stress increase deviation is similar to module $\mathrm{A}$. When a module with less noise is used it makes sense to increase the loop rate and achieve stress increase with smaller deviation from intended line.

The module A high uncertainty can be lowered by system calibration that is performed more frequently.

\section{CONCLUSION}

This paper presents the implementation of the virtual instrument for determination of uniaxial compression strength and deformability of rock samples, which performs the measurement and control of test.

The analysis of noise and error limits has shown that the properties of the first electronic component contribute most to the overall performance. Also, the analysis gives recommendation on how to improve the overall performance by using conditioning modules with better properties or by increasing the excitation voltage.

Usually, the properties of transducer are dominant in the measurement chain but it is shown here how the electronic part of the measurement system can substantially contribute to the overall performance, especially when small voltage ratio is measured.

The automatic control is achieved by using the described hardware and software. This allows fast and easy change in control function, which creates the opportunity to explore measurement methods for the determination of uniaxial compression strength.

\section{REFERENCES}

[1] European Committee for Standardization. (1999). European Standard: Natural Stone Test Methods Determination of Compressive Strength. EN 19261999. Brussels.

[2] Fairhurst, C.E., Hudson, J.A. (1999). Draft ISRM suggested method for the complete stress-strain curve for intact rock in uniaxial compression. International Society for Rock Mechanics and Mining Sciences, 36, 279-289.

[3] Godec, Z. (1995). Expression of Measurement Results. Zagreb, Croatia: Graphis. (In Croatian.)

[4] National Instruments Developer Zone. (2000) Using calibration to improve measurement accuracy. Retrieved April 2, 2007, from http://zone.ni.com/devzone/cda/tut/p/id/5188

[5] International Standardization Organization. (1995). Guide to the Expression of Uncertainty in Measurement. Geneva, Switzerland.

[6] National Instruments. (2008). DAQ M Series. User Manual. Retrieved February 9, 2009, from http://www.ni.com/pdf/manuals/371022k.pdf

Received April 20, 2011. Accepted August 3, 2011. 